\title{
DEPENDENCE OF THE VISCOSITY COEFFICIENT OF THE NIOSOMAL DISPERSION ON THE TEMPERATURE AND PARTICLE SIZE OF THE DISPERSED PHASE
}

\author{
Elena Igorevna Diskaeva ${ }^{a, *}$, Olga Vladimirovna Vecher ${ }^{a}$, \\ Igor Alexandrovich Bazikov ${ }^{b}$, Karine Sergeevna Elbekyan ${ }^{c}$, \\ Elena Nikolaevna Diskaeva ${ }^{d}$
}

a Stavropol State Medical University, Department of Physics and Mathematics, Mira 310, 355017 Stavropol, Russia

${ }^{b}$ Stavropol State Medical University, Department of Microbiology, Mira 310, 355017 Stavropol, Russia

c Stavropol State Medical University, Department of General and Biological Chemistry, Mira 310, 355017 Stavropol, Russia

${ }^{d}$ Branch of the Federal State Budget Educational Institution of Higher Education "MIREA - Russian Technological University" in Stavropol, Department of Industrial Technology, Kulakov Avenue 8, 355035 Stavropol, Russia

* corresponding author: e_diskaeva@mail.ru

\begin{abstract}
The aim of this study was to experimentally investigate the dependence of viscosity coefficient of niosomal dispersion based on PEG-12 Dimethicone on the temperature and size of niosomes vesicles. The experiments were carried out with niosomes, the average size of which varied from 85 to $125 \mathrm{~nm}$. The temperature varied from 20 to $60^{\circ} \mathrm{C}$, the volume concentration varied from 1 to $10 \%$. The particle size was determined by scanning electron microscopy (SEM) with subsequent statistical data processing. This study showed that the viscosity of niosomal dispersions significantly depends on both the temperature and the size of niosomes vesicles. With increasing temperature, the viscosity of niosomal dispersions decreases and with increasing particle size, the viscosity increases.
\end{abstract}

KEYWORDS: Niosome, nonionic surfactant vesicles, viscosity of niosomal dispersion, vesical size.

\section{INTRODUCTION}

The study of the physicochemical properties of niosomal dispersions is motivated by their wide application in pharmacology, medicine, and cosmetology 11. The optimal size of niosome vesicles for drug delivery systems is in a range (10-400 nm). Drug delivery systems often need nanoscale features, for example, less than $200 \mathrm{~nm}$ for parenteral and local transport into tissues, less than $300 \mathrm{~nm}$ for the eye chamber and less than 10 $\mathrm{nm}$ for the circulating bloodstream 244 .

Physicochemical characteristics of carrier particles determine their size, charge, elastic properties, ability to aggregate and deform, which affects biodistribution in the tissues, lifespan and elimination rate [5]7].

In most cases, the efficiency of using niosomal dispersions will significantly depend on the viscosity coefficient, since this coefficient determines the external energy required for organizing the flow and selecting the optimal dosage of drugs.

Almost all classical theories of viscosity believe that the viscosity coefficient is dependent only on the volume concentration, without taking into account its dependence on temperature, although, even for homogeneous liquids, this dependence is very significant 8 10.

Currently, there is a number of empirical and semiempirical formulas describing the changes in viscos- ity caused by temperature, which are applicable to determine the viscosity of dispersed systems. The more exactly these equations define the viscositytemperature dependence, the more coefficients they contain. However, the increased number of coefficients complicates the practical application of these formulas due to the need to experimentally determine each constant [11, 12.

The modified formula of Batschinsky formed the basis of the Slotte formula, which can be used for a quantitative description of the empirical dependence of viscosity on temperature [13]:

$$
\frac{c}{\eta}=(t+A)^{n}
$$

where $c, A$ and $n$ are constants, $t$ is temperature, $\eta$ is the coefficient of dynamic viscosity. The Bingham and Stokei formula is also often used:

$$
\frac{1}{\eta}=a T+b T^{2}
$$

where $a$ and $b$ are constants, $T$ is the absolute temperature.

There are other semi-empirical dependencies, but qualitatively, the dependence of the viscosity coefficient on temperature can be represented, in general 
form, by the expression:

$$
\eta=A \cdot \exp \left(\frac{B}{T}\right)
$$

where $A, B$ are constants.

However, formula (3) is used for a narrow temperature range. In the case of a sufficiently wide temperature range, they resort to formulas of other types, the most used of which is Vogel's formula:

$$
\eta=A \cdot \exp \left(\frac{B}{T-C}\right)
$$

where $A, B, C$ - are constants.

All considered dependencies are not universal and vary significantly depending on the concentration of the niosomal dispersion, the size of the niosomal vesicles, and the base fluid. The main contradictions arise with studying the dependence of viscosity on particle size. Many investigators assert that, as in ordinary coarsely dispersed liquids, this dependence is absent 14 .

It is worthy to note, that there is no common point of view regarding the dependence of the viscosity coefficient on temperature. Most researchers note a decrease in viscosity with increasing temperature. The situation is complicated by the fact that the dependence of the viscosity coefficient, even of ordinary dispersed liquids, on temperature has also not been properly studied.

The purpose of this work was to conduct an experimental study of the dependence of viscosity coefficient of a niosomal dispersion based on PEG-12 Dimethicone on particle size and temperature. The viscosity of niosomal dispersion was measured in a temperature interval $\left(20-60^{\circ} \mathrm{C}\right)$.

\section{MAterials AND METHOdS}

In our experiment, we used niosomes that consisted of a shell in the form of a water-insoluble double layer of a nonionic surfactant, which is a group of dimethiconecopolyol substances that are esters of polyethylene glycol and polydimethylsiloxane (PDMS) backbone [15-17].

To obtain the silicone-based capsules, physicochemical methods for the synthesis of molecules were used. In the hydrophilic part of dimethicone, there are functional groups of silicon oxide. The length of the Si-O bond was $1.6 \AA$, which is much longer than the $\mathrm{C}-\mathrm{C}$ bond of $1.4 \AA$. Due to this, the functional groups of the molecules are able to rotate with respect to each other. This provided the niosomes with a greater elasticity than that of liposomes made up of phospholipids. The use of PEG-12 Dimethicone promoted the formation of vesicles without requiring significant energy and effort. The Si-O-Si bond angle was 130 degrees, in contrast to the 109 degrees C-C-C bond, which increased the elasticity and stability [18.

The stage of formation of vesicles occurred with intensive mechanical mixing of the mixture using an
Silicon-based niosome

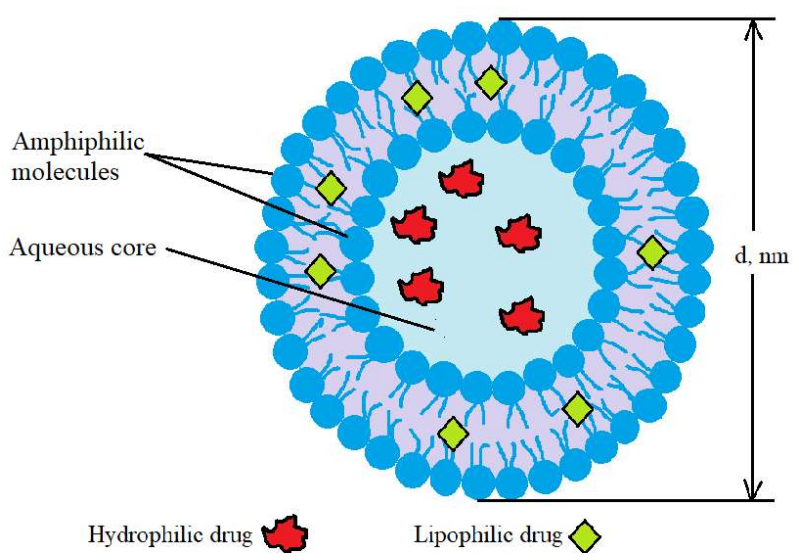

Figure 1. Structure of a silicone-based noisome.

automatic reclosure homogenizer at a room temperature for 5 minutes. The dispersion was then placed in a vessel for ultrasonic treatment. Ultrasonic sounding was carried out at a frequency of $20 \mathrm{kHz}$, power $200 \mathrm{~W}$, exposure -10 minutes. Then, to stabilize the concentration of hydrogen ions $(\mathrm{pH})$ to $6.6-7.0$ and the formation of a homogeneous structure of niosomes, emulsification was carried out on an APV homogenizer APV Lab Series Homogenizers - 1000.

Monolamellar niosomes with a mean vesicle size of $80-150 \mathrm{~nm}$ were formed. The prepared niosomes were spherical in shape. Then, the samples were diluted with ultrapure water.

A VPZh-1 capillary viscometer with a capillary diameter of $0.86 \mathrm{~mm}$ was used to measure the viscosity. To ensure measurements at a fixed temperature, the experiments were carried out using a thermostat with a fixation accuracy of $0.1^{\circ} \mathrm{C}$.

Since one of the tasks was to determine the dependence of the viscosity coefficient on the particle size, a high accuracy of determining the average size of niosomes and their size distribution was required. The dispersion of nanoparticles was studied using the scanning electron microscopic method (SEM Tescan Mira $3 \mathrm{Im})$. The sample preparation for SEM involved sample drying, mounting, and coating. Characteristics of the sample surface are obtained from the electrons emitted from the sample surface after the scanning with a focused electron beam.

The particle size, determined by Image J, MS Excel statistical package program, was used to perform the analysis 19 .

\section{Results And Discussion}

Niosomal dispersions with volumetric concentrations of 1,5 or $10 \%$ were selected for the research. The vesicle size analysis of the niosomal dispersion was carried out using scanning electron microscopy. A typical electron micrograph of niosomes vesicles with an average size of $103 \mathrm{~nm}$ is shown in Fig. 2. 


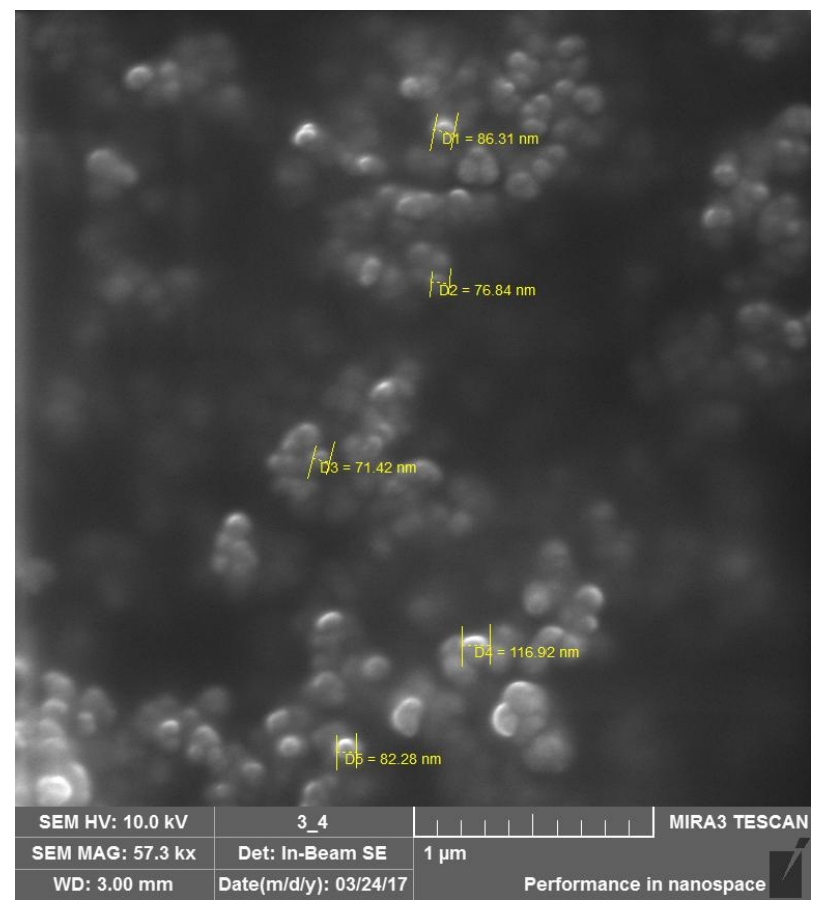

FiguRE 2. Micrograph of niosomes obtained by Scanning Electron Microscopy (SEM).

The microphotograph shows that niosomes, for the most part, are spherical particles. This implies that the projective diameter can be used for the equivalent diameter determination of the particles. The area of the project diameter will be equal to the area of the particle projection image.

Area of the projection of a spherical particle is equal to:

$$
S=\frac{\pi \delta_{p}^{2}}{4}
$$

Then, the average projection diameter can be presented in the form:

$$
\delta_{p}=\sqrt{\frac{4 S_{p}}{\pi}}
$$

To determine the average particle size for each volume concentration and to plot the particle size distribution, various fractions with a total number of particles of 500 were examined.

Particle probability density functions of the size of niosomes obtained by processing an ensemble of electron microphotographs are shown in Fig. 3

The graphs in Fig. 3 confirmed that in all cases, distributions are lognormal. A comparison of these values for different volume concentrations demonstrates homogenization with respect to particle size of the niosomes dispersion. Considering the interval of values of the vesicles diameter in the range from $85 \mathrm{~nm}$ to $130 \mathrm{~nm}$, it can be seen that higher volume concentration causes a decrease of particle size: from $123 \mathrm{~nm}$ at $1 \%$ to $92 \mathrm{~nm}$ at $10 \%$. The reason behind this phenomenon is, probably, the peculiarities of the intermolecular interaction of the vesicle shells and deformation of the vesicle membranes.
It should be noted that the complex nature of the mutual influence of such factors as the zeta potential, surface energy, and the distance between the shells complicates the construction of a mathematical model in a wide range of volume concentrations.

We assume that in more concentrated systems ( $>10 \%$ ), there will be a more intensive interaction between the particles of the dispersed phase, which may lead to the formation of temporary associates.

A further increase of concentration of the dispersed phase will lead to the formation of stable aggregates consisting of many particles. This will increase the viscosity of the system.

Fig. 4 describes the character of the dependence of viscosity on temperature for different volume concentrations.

Research results show that the dependence of the kinematic viscosity coefficient of dispersions is close to an exponential law with the coefficient increasing proportionally with an increase in the specific surface area of the dispersed phase.

A careful statistical analysis of experimental data revealed an exponential correlation given by (7), which fits the data with a correlation factor $R^{2}>0.99$.

$$
\nu A e^{-B T}
$$

Here, $\nu$ is the kinematic viscosity, $\mathrm{mm} / \mathrm{s}^{2} ; T$ is the temperature, ${ }^{\circ} \mathrm{C} ; A$ and $B$ are the functions of particle volume concentration $(\phi)$, the values of which are given in Table 1 .

The coefficient $A$ is related to the volume percentage given by

$$
A=37.75 \phi^{2}-0.8605 \phi+1.3707 \quad \text { with } R^{2}=0.995
$$

In the above expression, $\phi$ ranges from 1 to $10 \%$. The value of $B$ did not change. However, this does not exclude the possibility of its dependence on the volume concentration outside the range from 1 to $10 \%$.

Fig. 5 shows the experimental values of viscosity plotted against the size of niosomes.

Viscosity coefficients dispersions with different volume concentrations differ and grow with an increase in the diameter of niosomal vesicles. Presumably, this may be due to a decrease in the resistance at the phase "dispersion medium-niosome" interface due to a reduction in surface area 20 .

It can be concluded that the dependence of the viscosity coefficient of niosomal dispersions on particle size is rather complex and depends on the volume concentration of the dispersed phase [21-23.

Results obtained showed that not only the temperature but also the structural properties and the nature of intermolecular interactions have a noticeable effect on the viscosity (fluidity) of niosomal dispersions. 


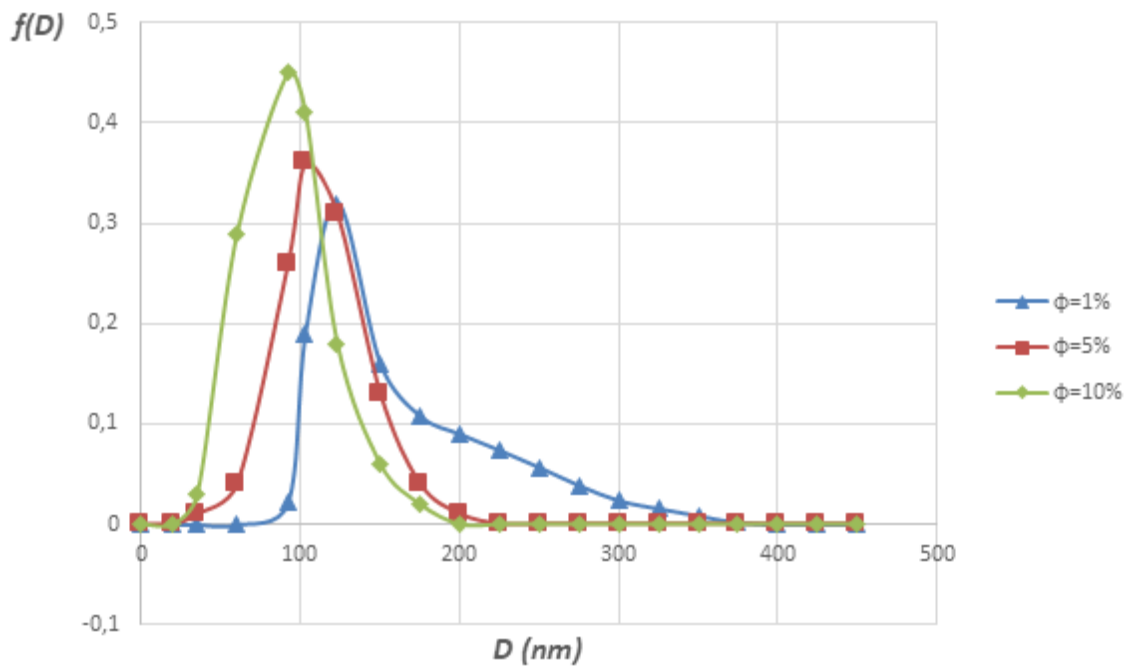

FIGURE 3. Probability density function of particle size distribution in niosomal dispersions for different volume concentration.

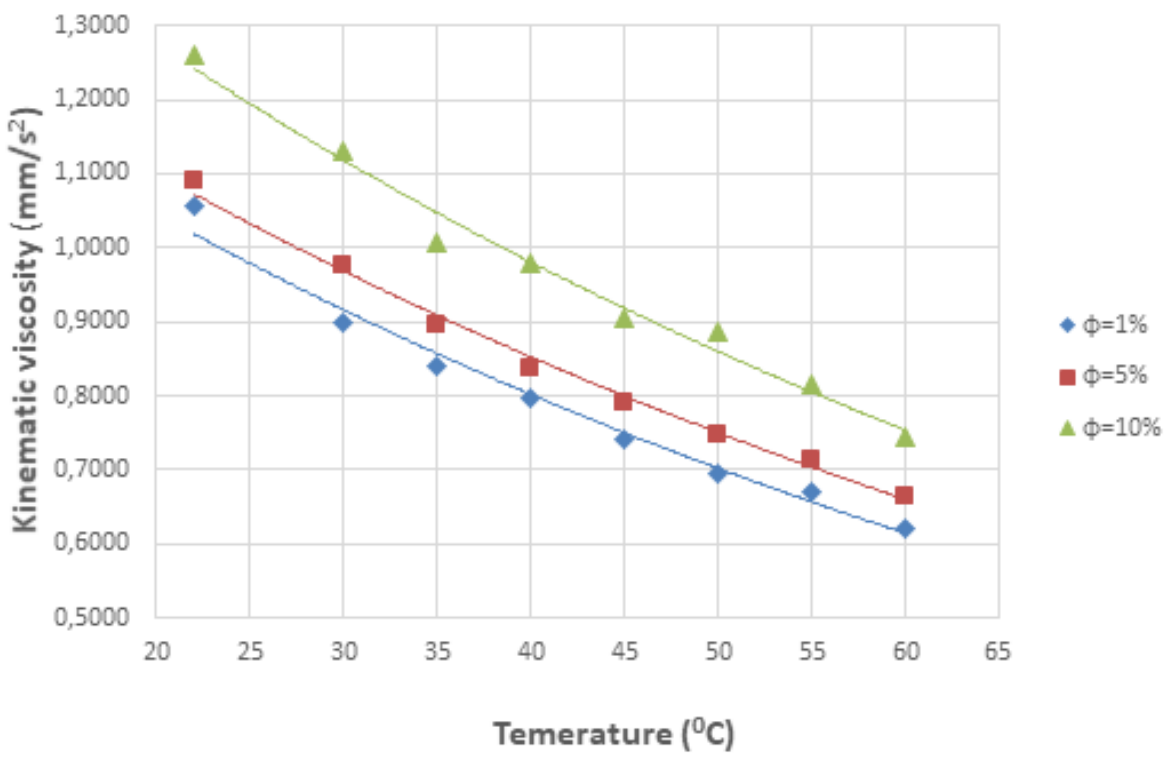

FIGURE 4. Change in viscosity with rise in temperature.

\begin{tabular}{cccc}
\hline Volume concentration $(\phi)$ & 0.01 & 0.05 & 0.10 \\
$A$ & 1.3658 & 1.4215 & 1.6601 \\
$B$ & 0.0130 & 0.0130 & 0.0130 \\
\hline
\end{tabular}

TABLE 1. Value of $A$ and $B$ with a correlation factor $R^{2}>0.99$. 


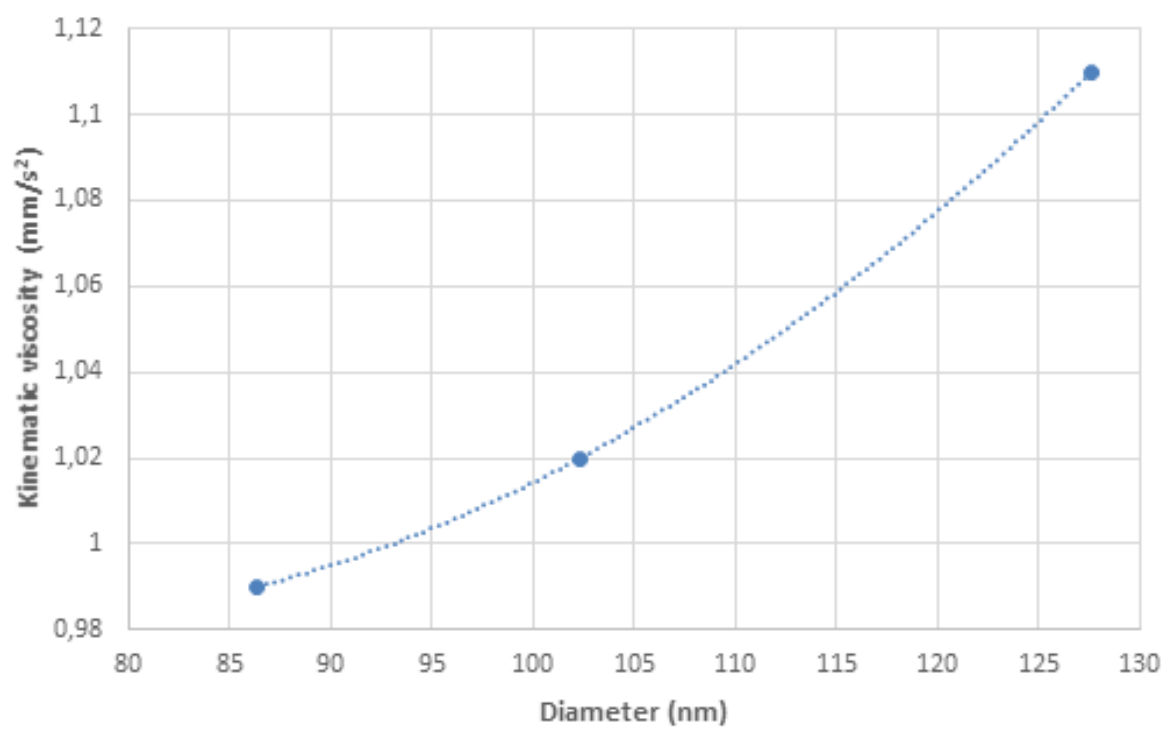

FiguRE 5. Dependence of viscosity plotted against the size of niosomes vesicles.

\section{Conclusion}

Niosomes are versatile drug delivery devices and have numerous therapeutic applications. Hence, this versatility necessitates proper physicochemical characterization techniques to suit the intended route of administration. The viscosity of niosomes is an important indication to evaluate the biodistribution in the tissues, lifespan and drug elimination rate.

This study shows that the viscosity of niosomes dispersion depends on many parameters, such as base fluid, particle volume fraction, particle size, temperature, particle size distribution and particle aggregation.

In this article, we attempted to study and characterise viscosity of a niosomal dispersion as the function of temperature and vesicle size.

Obtained results may give a better understanding of the residence time of drugs in the tissues and bioavailability products. Drug diffusion out of the formulation into the tissues may also be inhibited due to a high product viscosity. Finally, the administration of highviscosity liquid products tends to be more difficult.

\section{REFERENCES}

[1] R. C. Dutta. Drug carriers in pharmaceutical design: promises and progress. Current pharmaceutical design 13(7):761 - 769, 2007. https://doi.org/10.2174/138161207780249119.

[2] H. S. Nalwa (ed.). Handbook of nanostructured materials and nanotechnology. Academic Press, Boston, 2000 .

[3] S. P. Vyas, R. K. Khar. Targeted $\&$ controlled drug delivery : novel carrier systems. CBS Publishers \& Distributors, 2002.

[4] G. P. Kumar, P. Rajeshwarrao. Nonionic surfactant vesicular systems for effective drug delivery - an overview. Acta Pharmaceutica Sinica B 1(4):208 - 219, 2011. https://doi.org/10.1016/j.apsb.2011.09.002
[5] A. Kapoor. An overview on niosomes - A novel vesicular approach for ophthalmic drug delivery. Pharma Tutor 4(2):28 - 33, 2016.

[6] Z. S. Bayindir, N. Yuksel. Characterization of niosomes prepared with various nonionic surfactants for paclitaxel oral delivery. Journal of Pharmaceutical Sciences 99(4):2049 - 2060, 2010. https://doi.org/10.1002/jps.21944

[7] V. F. Naggar, S. S. El gamal, A. N. Allam. Proniosomes as a stable carrier for oral Acyclovir: Formulation and physicochemical characterization. Journal of American Science 8(9):417 - 428, 2012.

[8] R. Pal. Modeling the viscosity of concentrated nanoemulsions and nanosuspensions. Fluids 1(2), 2016. https://doi.org/10.3390/fluids1020011

[9] J. Happel, H. Brenner. The Viscosity of Particulate Systems, pp. 431 - 473. Springer Netherlands, Dordrecht, 1983. https://doi.org/10.1007/978-94-009-8352-6_9.

[10] J. Gonzalez-Gutierrez, S. Hert, B. von Bernstorff, I. Emri. Prediction of viscosity of pim feedstock materials with different particle size distribution. In 31th Danubia-Adria Symposium in Advances in Experimental Mechanics. Kempten University, Germany, 2014.

[11] H. J. H. Brouwers. Viscosity of a concentrated suspension of rigid monosized particles. Physical Review E 81(5):051402, 2010. https://doi.org/10.1103/PhysRevE.81.051402

[12] M. Ochowiak, J. Różański. Rheology and structure of emulsions and suspensions. Journal of Dispersion Science and Technology 33(2):177 - 184, 2012. https://doi.org/10.1080/01932691.2010.548694.

[13] A. J. Batschinski. Untersuchungen Aber die innere Reibnng der Flüssigkeiten. I. Zeitschrift für Physikalische Chemie 84(1):643 - 706, 1913. https://doi.org/10.1515/zpch-1913-8442

[14] S. Mueller, E. Llewellin, H. Mader, et al. The rheology of suspensions of solid particles. Proceedings of The Royal Society A: Mathematical, Physical and Engineering Sciences 466(2116):1201 - 1228, 2009. https://doi.org/10.1098/rspa.2009.0445 
[15] I. A. Bazikov, P. A. Omelyanchuk. The method of delivery of biologically active substances with the help of niosomes, RF patent, 2539396, 2014.

[16] I. A. Bazikov. A method for transdermal transfer of active substances using niosomes on the basis of PEG-12 dimethicone, RF patent, 2320323, 2008.

[17] I. A. Bazikov, V. V. Lukinova, A. N. Maltsev, et al. Interaction niosomal doxorubicin cell membranes. Medical Gazette of the North Caucasus 11(1):108 - 110, 2016. https://doi.org/10.14300/mnnc.2016.11011

[18] I. A. Bazikov, V. V. Lukinova, N. I. Malinina, A. N. Maltsev. Study of the mechanisms of intercellular interaction of the niosomal form of the antitumor drug doxorubicin with plasma membranes. The Eurasian Union of Scientists 3(24):34, 2016.

[19] E. I. Diskaeva, O. V. Vecher, I. A. Bazikov, D. S. Vakalov. Particle size analysis of niosomes as a function of temperature. Nanosystems: physics, chemistry, mathematics 9(2):290- 294, 2018. https://doi.org/10.17586/2220-8054-2018-9-2-290-294.
[20] V. Y. Rudyak, A. A. Belkin, V. V. Egorov. Effective viscosity coefficient of nanosuspensions. In AIP Conference Proceedings, vol. 1084, pp. 489 - 494. 2008. https://doi.org/10.1063/1.3076527

[21] M. N. Yasin, S. Hussain, F. Malik, et al. Preparation and characterization of chloramphenicol niosomes and comparison with chloramphenicol eye drops $(0.5 \% \mathrm{w} / \mathrm{v})$ in experimental conjunctivitis in albino rabbits. Pakistan journal of pharmaceutical sciences 25(1):117 - 121, 2012.

[22] S. B. Shirsand, G. R. Kumar, G. G. Keshavshetti, et al. Formulation and evaluation of clotrimazole niosomal gel for topical application. Rajiv Gandhi University of Health Sciences Journal of Pharmaceutical Sciences 5(1):32 - 38, 2015.

[23] B. Lu, Y. Huang, Z. Chen, et al. Niosomal nanocarriers for enhanced skin delivery of quercetin with functions of anti-tyrosinase and antioxidant. Molecules 24(12):2322, 2019.

https://doi.org/10.3390/molecules24122322 